\title{
A Comparative Study of Information-gathering Approaches for Answering Help-desk Email Inquiries
}

\author{
Ingrid Zukerman and Yuval Marom \\ Faculty of Information Technology, Monash University \\ Clayton, Victoria 3800, AUSTRALIA \\ \{ingrid,yuvalm\}@csse.monash.edu.au
}

\begin{abstract}
We present a comparative study of corpus-based methods for the automatic synthesis of email responses to help-desk requests. Our methods were developed by considering two operational dimensions: (1) information-gathering technique, and (2) granularity of the information. In particular, we investigate two techniques - retrieval and prediction - applied to information represented at two levels of granularity - sentence level and document level. We also developed a hybrid method that combines prediction with retrieval. Our results show that the different approaches are applicable in different situations, addressing a combined $72 \%$ of the requests with either complete or partial responses.
\end{abstract}

\section{Introduction}

Email inquiries sent to help desks often "revolve around a small set of common questions and issues". This means that help-desk operators spend most of their time dealing with problems that have been previously addressed. Further, a significant proportion of help-desk responses contain a low level of technical content, corresponding, for example, to inquiries addressed to the wrong group, or insufficient detail provided by the customer about his or her problem. Organizations and clients would benefit if the efforts of human operators were focused on difficult, atypical problems, and an automated process was employed to deal with the easier problems.

In this paper, we report on our experiments with corpus-based approaches to the automation of help-desk responses. Our study was based on a log of 30,000 email dialogues between users and help-desk operators at Hewlett-Packard. However, to focus our work, we used a sub-corpus of 6659 email dialogues, which consisted of two-turn dialogues where the answers were reasonably concise (15 lines at most). These dialogues deal with a variety of user requests, which include requests for technical assistance, inquiries about products, and queries about how to return faulty products or parts. As a first step, we have automatically clustered the corpus according to the subject line of the first email. This process yielded 15 topic-based datasets that contain between 135 and 1200 email dialogues. Owing to time limitations, the procedures described in this paper were applied to 8 of the datasets, corresponding to approximately $75 \%$ of the dialogues.

Analysis of our corpus yields the following observations:

\footnotetext{
${ }^{1}$ http://customercare.telephonyonline.com/ar/telecom_next_ generation_customer.
} 


\section{RA1:}

Do I need Compaq driver software for my armada 1500 docking station? This in order to be able to re-install win 98 ?

I would recommend to install the latest system rompaq, on the laptop and the docking station. Just select the model of computer and the operating system you have. http:// www. thislink. com.

\section{RA2:}

Is there a way to disable the NAT firewall on the Compaq CP-2W so I don't get a private ip address through the wireless network?

Unfortunately, you have reached the incorrect eResponse queue for your unit. Your device is supported at the following link, or at 888-phone-number. We apologize for the inconvenience.

Fig. 1. Sample request-answer pairs.

- 01: Requests containing precise information, such as product names or part specifications, sometimes elicit helpful, precise answers referring to this information, while other times they elicit answers that do not refer to the query terms, but contain generic information (e.g., referring customers to another help group or asking them to call a particular phone number). Request-answer pair RA1 in Figure 1 illustrates the first situation, while the pair RA2 illustrates the second. ${ }^{2}$

- 02: Operators tend to re-use the same sentences in different responses. This is partly a result of companies having in-house manuals that prescribe how to generate an answer. For instance, answers A3 and A4 in Figure 2 share the sentence in italics.

These observations prompt us to consider complementary approaches along two separate dimensions of our problem. The first dimension pertains to the technique applied to determine the information in an answer, and the second dimension pertains to the granularity of the information.

Observation 01 leads us to consider two techniques for obtaining information: retrieval and prediction. Retrieval returns an information item by matching its terms to query terms [1]. Hence, it is likely to obtain precise information if available. In contrast, prediction uses correlations between features of requests and responses to select an information item. For example, the absence of a particular term in a request may be a good predictive feature (which cannot be considered in traditional retrieval). Thus, prediction could yield replies that do not match particular query terms.

Observation $\mathbf{O 2}$ leads us to consider two levels of granularity: document and sentence. That is, we can obtain a document comprising a complete answer on the basis of a request (i.e., re-use an answer to a previous request), or we can obtain individual sentences and then combine them to compose an answer, as is done in multi-document summarization [2]. The sentence-level granularity enables the re-use of a sentence for different responses, as well as the composition of partial responses.

The methods developed on the basis of these two dimensions are: Retrieve Answer, Predict Answer, Predict Sentences, Retrieve Sentences and Hybrid Predict-Retrieve Sentences. The first four methods represent the possible combinations of information-

\footnotetext{
${ }^{2}$ Our examples are reproduced verbatim from the corpus (except for URLs and phone numbers which have been disguised by us), and some have user or operator errors.
} 
A3:

If you are able to see the Internet then it sounds like it is working, you may want to get in touch with your IT department to see if you need to make any changes to your settings to get it to work. Try performing a soft reset, by pressing the stylus pen in the small hole on the bottom left hand side of the Ipaq and then release.

A4:

I would recommend doing a soft reset by pressing the stylus pen in the small hole on the left hand side of the Ipaq and then release. Then charge the unit overnight to make sure it has been long enough and then see what happens. If the battery is not charging then the unit will need to be sent in for repair.

Fig. 2. Sample answers that share a sentence.

gathering technique and level of granularity; the fifth method is a hybrid where the two information-gathering techniques are applied at the sentence level.

Our aim in this paper is to investigate when the different methods are applicable, and whether individual methods are uniquely successful in certain situations. For this purpose, we decided to assign a level of success not only to complete responses, but also to partial ones (obtained with the sentence-based methods). The rationale for this is that we believe that a partial high-precision response is better than no response, and better than a complete response that contains incorrect information. We plan to test these assumptions in future user studies.

The rest of this paper is organized as follows. In the next section, we describe our five methods, followed by the evaluation of their results. In Section 4, we discuss related research, and then present our conclusions and plans for future work.

\section{Information-gathering Methods}

In the following sub-sections we present the implementation details of the various methods. Note that some of the methods were implemented independently of each other at different stages of our project. Consequently, there are minor implementational variations, such as choice of machine learning algorithms and some discrepancy regarding features. We plan to bridge these differences in the near future, but are confident that they do not impede the aim of the current study: exploring the performance of our system along the two dimensions mentioned in the previous section (information-gathering approach, and level of granularity).

\subsection{Retrieve a Complete Answer}

This method retrieves a complete document (answer) on the basis of request lemmas. We use cosine similarity to determine a retrieval score, and use a minimal retrieval threshold that must be surpassed for a response to be accepted.

We have considered three approaches to indexing the answers in our corpus: according to the content lemmas in (1) requests, (2) answers, or (3) requests\&answers. The results in Section 3 are for the third approach, which proved best. To illustrate the difference between these approaches, consider request-answer pair RA2. If we received a new request similar to that in RA2, the answer in RA2 would be retrieved if we had indexed according to requests or requests\&answers. However, if we had indexed only on answers, then the response would not be retrieved. 


\subsection{Predict a Complete Answer}

This prediction method first groups similar answers in the corpus into answer clusters. For each request, we then predict an answer cluster on the basis of the request features, and select the answer that is most representative of the cluster (closest to the centroid). This method would predict a group of answers similar to the answer in RA2 from the input lemmas "compaq" and "cp-2w".

The clustering is performed in advance of the prediction process by the clustering program Snob [3], using the content lemmas (unigrams) in the answers as features. The predictive model is a Decision Graph [4] trained on (1) input features: lemma unigrams and bigrams in the request, ${ }^{3}$ and (2) target feature - the identifier of the answer cluster that contains the actual answer for the request. The model provides a prediction of which response cluster is most suitable for a given request, as well as a level of confidence in this prediction. We do not attempt to produce an answer if the confidence is not sufficiently high.

In principle, rather than clustering the answers, the predictive model could have been trained on individual answers. However, on one hand, the dimensionality of this task is very high, and on the other hand, answers that share significant features would be predicted together, effectively acting as a cluster. By clustering answers in advance, we reduce the dimensionality of the problem, at the expense of some loss of information (since somewhat dissimilar answers may be grouped together).

\subsection{Predict Sentences}

This method looks at each answer sentence as though it were a separate document, and groups similar sentences into clusters in order to obtain meaningful sentence abstractions and avoid redundancy. ${ }^{4}$ For instance, the last sentence in $\mathbf{A 3}$ and the first sentence in A4 are assigned to the same sentence cluster. As for Answer Prediction (Section 2.2), this clustering process also reduces the dimensionality of the problem.

Each request is used to predict promising clusters of answer sentences, and an answer is composed by extracting a sentence from such clusters. Because the sentences in each cluster originate from different response documents, the process of selecting them for a new response corresponds to multi-document summarization. In fact, our selection mechanism, described in more detail in [6], is based on a multi-document summarization formulation proposed by Filatova and Hatzivassiloglou [2].

In order to be able to generate appropriate answers in this manner, the sentence clusters should be cohesive, and they should be predicted with high confidence. A cluster is cohesive if the sentences in it are similar to each other. This means that it is possible to obtain a sentence that represents the cluster adequately (which is not the case for an uncohesive cluster). A high-confidence prediction indicates that the cluster is relevant to many requests that share certain regularities. Owing to these requirements, the Sentence Prediction method will often produce partial answers (i.e., it will have a high precision, but often a low recall).

\footnotetext{
${ }^{3}$ Significant bigrams are obtained using the NSP package [5].

${ }^{4}$ We did not cluster request sentences, as requests are often ungrammatical, which makes it hard to segment them into sentences, and the language used in requests is more diverse than the corporate language used in responses.
} 
Sentence clustering. The clustering is performed by applying Snob using the content lemmas (unigrams) in the sentences as features, similarly to the answer clustering in the Predict Answer method (Section 2.2). ${ }^{5}$

Calculation of cluster cohesion. Our cohesion measure implements the idea that a cohesive group of sentences should agree strongly on both the words that appear in these sentences and the words that are omitted. Hence, it is possible to obtain a sentence that adequately represents a cohesive sentence cluster, while this is not the case for a loose sentence cluster. For example, the italicized sentences in A3 and A4 belong to a highly cohesive sentence cluster (0.93), while the opening answer sentence in RA1 belongs to a less cohesive cluster (0.7) that contains diverse sentences about the Rompaq power management. Our measure is similar to entropy, in the sense that it yields non-zero values for extreme probabilities [6].

Sentence-cluster prediction. We use a Support Vector Machine (SVM) for predicting sentence clusters. A separate SVM is trained for each sentence cluster, with lemma unigrams and bigrams in a request as input features, and a binary target feature specifying whether the cluster contains a sentence from the response to this request. During the prediction stage, the SVMs predict zero or more clusters for each request. One representative sentence (closest to the centroid) is then extracted from each highly cohesive cluster predicted with high confidence. These sentences will appear in the answer (at present, these sentences are treated as a set, and are not organized into a coherent reply).

\subsection{Retrieve Sentences}

As for Sentence Prediction (Section 2.3), this method looks at each answer sentence as though it were a separate document. For each request sentence, we retrieve candidate answer sentences on the basis of the match between the content lemmas in the request sentence and the answer sentence. For example, while the first answer sentence in RA1 might match the first request sentence in RA1, an answer sentence from a different response (about re-installing Win98) might match the second request sentence.

We are mainly interested in answer sentences that "cover" request sentences, i.e., the terms in the request should appear in the answer. Hence, we use recall as the measure for the goodness of a match, where recall is defined as follows.

$$
\text { recall }=\frac{\text { TF.IDF of lemmas in request sent } \& \text { answer sent }}{\text { TF.IDF of lemmas in request sentence }}
$$

Initially, we retain the answer sentences whose recall exceeds a threshold. ${ }^{6}$ Once we have the set of candidate answer sentences, we attempt to remove redundant sentences. This requires the identification of sentences that are similar to each other - a task for which we use the sentence clusters described in Section 2.3. Given a group of answer sentences that belong to the same cohesive cluster, we retain the sentence with the highest recall (in our current trials, a cluster is sufficiently cohesive for this purpose if its cohesion $\geq 0.7$ ). In addition, we retain all the answer sentences that do not belong to a cohesive cluster. All the retained sentences will appear in the answer.

\footnotetext{
${ }^{5}$ We have also tried syntactic features, but they did not produce superior results.

${ }^{6}$ To assess the goodness of a sentence, we experimented with $f$-scores that had different weights for recall and precision. Our results were insensitive to these variations.
} 


\subsection{Hybrid Predict-Retrieve Sentences}

It is possible that the Sentence Prediction method predicts a sentence cluster that is not sufficiently cohesive for a confident selection of a representative sentence. However, a sentence can still be selected by using clues from the request. For example, selecting between a group of sentences concerning the installation of different drivers might be possible if the request mentions a specific driver. Thus the Sentence Prediction method is complemented with the Sentence Retrieval method to form a hybrid, as follows.

- For highly cohesive clusters predicted with high confidence, we select a representative sentence as before.

- For clusters with medium cohesion predicted with high confidence, we attempt to match the sentences with the request sentences, using the Sentence Retrieval method but with a lower recall threshold. This reduction takes place because the high prediction confidence provides a guarantee that the sentences in the cluster are suitable for the request, so there is no need for a conservative recall threshold. The role of retrieval is now to select the sentence whose content lemmas best match the request, regardless of how good this match is.

- For uncohesive clusters or clusters predicted with low confidence, we have to resort to word matches, which means reverting to the higher, more conservative recall threshold, because we no longer have the prediction confidence.

\section{Evaluation}

As mentioned in Section 1, our corpus was divided into topic-based datasets. We have observed that the different datasets lend themselves differently to the various informationgathering methods described in the previous section. In this section, we examine the overall performance of the five methods across the corpus, as well as their performance for different datasets.

\subsection{Measures}

We are interested in two performance indicators: coverage and quality.

Coverage is the proportion of requests for which a response can be generated. The various information gathering methods presented in the previous section have acceptance criteria that indicate that there is some level of confidence in generating a response. A request for which a planned response fails to meet these criteria is not covered, or addressed, by the system. We are interested in seeing if the different methods are applicable in different situations, that is, how exclusively they address different requests. Note that the sentence-based methods generate partial responses, which are considered acceptable so long as they contain at least one sentence generated with high confidence. In many cases these methods produce obvious and non-informative sentences such as "Thank you for contacting HP", which would be deemed an acceptable response. We have manually excluded such sentences from the calculation of coverage, in order to have a more informative comparison between the different methods.

Ideally, the quality of the generated responses should be measured through a user study, where people judge the correctness and appropriateness of answers generated 
by the different methods. However, we intend to refine our methods further before we conduct such a study. Hence, at present we rely on a text-based quantitative measure. Our experimental setup involves a standard 10-fold validation procedure, where we repeatedly train on $90 \%$ of a dataset and test on the remaining $10 \%$. We then evaluate the quality of the answers generated for the requests in each test split, by comparing them with the actual responses given by the help-desk operator for these requests.

We are interested in two quality measures: (1) the precision of a generated response, and (2) its overall similarity to the actual response. The reason for this distinction is that the former does not penalize for a low recall - it simply measures how correct the generated text is. As stated in Section 1, a partial but correct response may be better than a complete response that contains incorrect units of information. However, more complete responses are generally better than partial ones, and so we use the second measure to get an overall indication of how correct and complete a response is. We use the traditional Information Retrieval precision and f-score measures [1], employed on a word-by-word basis, to evaluate the quality of the generated responses. ${ }^{7}$

\subsection{Results}

Table 1 shows the overall results obtained using the different methods. We see that combined the different methods can address $72 \%$ of the requests. That is, at least one of these methods can produce some non-empty response to $72 \%$ of the requests. Looking at the individual coverages of the different methods we see that they must be applicable in different situations, because the highest individual coverage is $43 \%$.

The Answer Retrieval method addresses $43 \%$ of the requests, and in fact, about half of these $(22 \%)$ are uniquely addressed by this method. However, in terms of the quality of the generated response, we see that the performance is very poor (both precision and f-score have very low averages). Nevertheless, there are some cases where this method uniquely addresses requests quite well.

The Answer Prediction method can address $29 \%$ of the requests. Only about a tenth of these are uniquely addressed by this method, but the generated responses are of a fairly high quality, with an average precision and f-score of 0.82 . Notice the large standard deviation of these averages, suggesting a somewhat inconsistent behaviour. This is due to the fact that this method gives good results only when complete generic responses are found. In this case, any re-used response will have a high similarity to the actual response. However, when this is not the case, the performance degrades substantially, resulting in inconsistent behaviour. This means that Answer Prediction is suitable when requests that share some regularity receive a complete template answer.

The Sentence Prediction method can find regularities at the sub-document level, and therefore deal with cases where partial responses can be generated. It produces responses for $34 \%$ of the requests, and does so with a consistently high precision (average 0.94 , standard deviation 0.13 ). Only an overall $1 \%$ of the requests are uniquely addressed by this method. However, for the cases that are shared between this method and other ones, it is useful to compare the actual quality of the generated response. In $5 \%$ of the cases, the Sentence Prediction method either uniquely addresses requests, or

\footnotetext{
${ }^{7}$ We have also employed sequence-based measures using the ROUGE tool set [7], with similar results to those obtained with the word-by-word measure.
} 
Table 1. Performance of the different methods, measured as coverage, precision and f-score.

\begin{tabular}{|l|c|c|c|}
\hline \multicolumn{1}{|c|}{ Method } & Coverage & Precision Ave (stdev) & F-score Ave (stdev) \\
\hline Answer Retrieval & $43 \%$ & $0.37(0.34)$ & $0.35(0.33)$ \\
Answer Prediction & $29 \%$ & $0.82(0.21)$ & $0.82(0.24)$ \\
Sentence Prediction & $34 \%$ & $0.94(0.13)$ & $0.78(0.18)$ \\
Sentence Retrieval & $9 \%$ & $0.19(0.19)$ & $0.12(0.11)$ \\
Sentence Hybrid & $43 \%$ & $0.81(0.29)$ & $0.66(0.25)$ \\
\hline Combined & $72 \%$ & $0.80(0.25)$ & $0.50(0.33)$ \\
\hline
\end{tabular}

jointly addresses requests together with other methods but has a higher f-score. This means that in some cases a partial response has a higher quality than a complete one.

Like the document-level Answer Retrieval method, the Sentence Retrieval method performs poorly. It is difficult to find an answer sentence that closely matches a request sentence, and even when this is possible, the selected sentences tend to be different to the ones used by the help-desk operators, hence the low precision and f-score. This is discussed further below in the context of the Sentence Hybrid method.

The Sentence Hybrid method extends the Sentence Prediction method by employing sentence retrieval as well, and thus has a higher coverage $(45 \%)$. In fact, the retrieval component serves to disambiguate between groups of candidate sentences, thus enabling more sentences to be included in a generated response. This, however, is at the expense of precision, as we also saw for the pure Sentence Retrieval method. Although retrieval selects sentences that match closely a given request, this selection can differ from the "selections" made by the operator in the actual response. Precision (and hence f-score) penalizes such sentences, even when they are more appropriate than those in the model response. For example, consider request-answer pair RA5. The answer is quite generic, and is used almost identically for several other requests. The Hybrid method almost reproduces this answer, replacing the first sentence with A6. This sentence, which matches more request words than the first sentence in the model answer, was selected from a sentence cluster that is not highly cohesive, and contains sentences that describe different reasons for setting up a repair (the matching word in A6 is "screen"). Overall, the Hybrid method outperforms the other methods in about $10 \%$ of the cases, where it either uniquely addresses requests, or addresses them jointly with other methods but produces responses with a higher f-score.

\begin{tabular}{l}
\hline RA5: \\
My screen is coming up reversed (mirrored). There must be something loose electronically \\
because if I put the stylus in it's hole and move it back and forth, I can get the screen to \\
display properly momentarily. Please advise where to send for repairs. \\
To get the iPAQ serviced, you can call $1-800-$ phone-number, options 3,1 (enter a 10 \\
digit phone number), 2. Enter your phone number twice and then wait for the routing center \\
to put you through to a technician with Technical Support. They can get the unit picked up \\
and brought to our service center. \\
$\begin{array}{l}\text { A6: } \\
\text { To get the iPAQ repaired (battery, stylus lock and screen), please call } \\
1-800-\text { phone-number, options 3, (enter a } 10 \text { digit phone number), 2. }\end{array}$
\end{tabular}




\subsection{Summary}

In summary, our results show that, with the exception of the Sentence Retrieval method, the different methods are applicable in different situations, all occurring significantly in the corpus. The Answer Retrieval method uniquely addresses a large portion of the requests, but many of its attempts are spurious, thus lowering the combined overall quality shown at the bottom of Table 1 (average f-score 0.50 ), calculated by using the best performing method for each request. The Answer Prediction method is good at addressing situations that warrant complete template responses. However, its confidence criteria might need refining to lower the variability in quality. The combined contribution of the sentence-based methods is substantial (about 15\%), suggesting that partial responses of high precision may be better than complete responses with a lower precision.

\section{Related Research}

There are very few reported attempts at corpus-based automation of help-desk responses. The retrieval system eResponder [8] retrieves a list of request-response pairs and presents a ranked list of responses to the user. In contrast, our system can re-use a single representative response, or collate sentences from multiple responses to generate a single (possibly partial) response. Lapalme and Kosseim [9] employ a rule-based, templatebased system that extracts patterns from a corpus of request-response emails in order to personalize a response (for example substituting names of individuals and companies). They also employ a retrieval component that finds answers from a corpus of technical documents (not the request-response corpus). Our work differs from these two examples in that it applies a purely data-driven approach that uses a corpus of request-response pairs to generate a single response. Similar corpus-based approaches have been implemented in FAQ domains $[10,11]$. However, this kind of corpus is significantly different to ours in that it lacks repetition and redundancy and the responses are not personalized.

\section{Conclusion and Future Work}

We have presented four basic methods and one hybrid method for addressing helpdesk requests. The basic methods represent the four ways of combining level of granularity (sentence and document) with information-gathering technique (prediction and retrieval). The hybrid method applies prediction possibly followed by retrieval to information at the sentence level. Our results show that with the exception of Sentence Retrieval, the different methods can address a significant portion of the requests. A future avenue of research is thus to characterize situations where different methods are applicable, in order to derive decision procedures that determine the best method automatically. We have also started to investigate an intermediate level of granularity: paragraphs.

Our results suggest that the automatic evaluation method requires further consideration. As seen in Section 3, our f-score penalizes the Sentence Prediction and Hybrid methods when they produce good answers that are more informative than the model answer. As mentioned previously, a user study would provide a more conclusive evaluation of the system, and could be used to determine preferences regarding partial responses. 
Finally, we propose the following extensions to our current implementation. First, we would like to improve the representation used for clustering, prediction and retrieval by using features that incorporate word-based similarity metrics [12]. Secondly, we intend to investigate a more focused sentence retrieval approach that utilizes syntactic matching of sentences. For example, if a sentence cluster is strongly predicted by a request, but the cluster is uncohesive because of a low verb agreement, then the retrieval should favour the sentences whose verbs match those in the request.

\section{Acknowledgments}

This research was supported in part by grant LP0347470 from the Australian Research Council and by an endowment from Hewlett-Packard. The authors also thank HewlettPackard for the extensive help-desk data.

\section{References}

1. Salton, G., McGill, M.: An Introduction to Modern Information Retrieval. McGraw Hill (1983)

2. Filatova, E., Hatzivassiloglou, V.: A formal model for information selection in multisentence text extraction. In: COLING'04 - Proceedings of the 20th International Conference on Computational Linguistics, Geneva, Switzerland (2004) 397-403

3. Wallace, C., Boulton, D.: An information measure for classification. The Computer Journal 11(2) (1968) 185-194

4. Oliver, J.: Decision graphs - an extension of decision trees. In: Proceedings of the Fourth International Workshop on Artificial Intelligence and Statistics, Fort Lauderdale, Florida (1993) 343-350

5. Banerjee, S., Pedersen, T.: The design, implementation, and use of the Ngram Statistics Package. In: Proceedings of the Fourth International Conference on Intelligent Text Processing and Computational Linguistics, Mexico City (2003)

6. Marom, Y., Zukerman, I.: Towards a framework for collating help-desk responses from multiple documents. In: Proceedings of the IJCAI05 Workshop on Knowledge and Reasoning for Answering Questions, Edinburgh, Scotland (2005) 32-39

7. Lin, C., Hovy, E.: Automatic evaluation of summaries using n-gram co-occurrence statistics. In: Proceedings of the 2003 Language Technology Conference (HLT-NAACL 2003), Edmonton, Canada (2003)

8. Carmel, D., Shtalhaim, M., Soffer, A.: eResponder: Electronic question responder. In: CoopIS '02: Proceedings of the 7th International Conference on Cooperative Information Systems, Eilat, Israel (2000) 150-161

9. Lapalme, G., Kosseim, L.: Mercure: Towards an automatic e-mail follow-up system. IEEE Computational Intelligence Bulletin 2(1) (2003) 14-18

10. Berger, A., Mittal, V.: Query-relevant summarization using FAQs. In: ACL2000 - Proceedings of the 38th Annual Meeting of the Association for Computational Linguistics, Hong Kong (2000) 294-301

11. Soricut, R., Brill, E.: Automatic question answering using the web: Beyond the factoid. Information Retrieval 9(2) (2006) 191-206

12. Pedersen, T., Patwardhan, S., Michelizzi, J.: WordNet::Similarity - measuring the relatedness of concepts. In: AAAI-04 - Proceedings of the Nineteenth National Conference on Artificial Intelligence, San Jose, California (2004) 25-29 